\title{
Perceived social and built environment associations of leisure-time physical activity among adults in Sri Lanka
}

Shreenika De Silva Weliange ${ }^{\text {*}^{*}}$, Madhawa Perera ${ }^{2}$ and Jagath Gunatilake $\mathrm{M}^{3,4}$

\begin{abstract}
Objective: Although perceived neighbourhood environment is considered a predictor of leisure-time physical activity (LTPA), evidence for this is limited in South Asia. Thus, the aim was to determine the association between neighbourhood social and built environment features in carrying out LTPA among adults in Colombo District, Sri Lanka. A cross-sectional study among 1320 adults was carried out using validated questionnaires for physical activity (PA) and built environment data collection. Multiple logistic regression analysis was conducted to assess the associations between environment characteristics and LTPA after adjusting for gender, age, employment status, income level and sector of residence.

Results: A total of $21.7 \%$ of adults participated in some LTPA. The commonest type of LTPA was walking; carried out by $14.5 \%$. Moderate and vigorous activity at leisure was carried out by $10.3 \%$ and $3.9 \%$ respectively. Perceived social acceptance for PA was positively associated with LTPA. Out of the built environment characteristics perceived infrastructure for walking, and recreational facilities for PA were negatively associated with LTPA. Self-efficacy emerged as an important positive correlate of LTPA. The participants were positively influenced by the self-efficacy and perceived social environment which should be addressed when promoting LTPA.
\end{abstract}

Keywords: Leisure time physical activity, Social environment, Built environment, Self-efficacy

\section{Introduction}

Physical activity (PA) reduces the risk of non-communicable diseases (NCDs), premature mortality and increase life expectancy [1]. Although PA levels of South Asian adults are modest, participation in leisure-time physical activity (LTPA) is not sufficient [2]. This low level of LTPA was also observed in Sri Lanka [3], and requires immediate attention in order to reduce the burden of NCDs, as domestic and job-related PA has also been rapidly reducing due to urbanised lifestyles $[2,3]$.

\footnotetext{
*Correspondence: shreenika@commed.cmb.ac.lk

${ }^{1}$ Department of Community Medicine, University of Colombo, Colombo, Sri Lanka

Full list of author information is available at the end of the article
}

Ecological approach is better suited to create active living communities as people select different places for LTPA depending on the built and social environment features [4]. Ecological model of PA describes the importance of multilevel approaches targeting individuals, social and built environments to promote PA at population level by providing safe, attractive, and convenient places, encouraging the use of those places, and changing social norms or culture [4]. Features of the built environment like walkable community designs, aesthetics, pedestrian facilities, bicycle facilities, trails and parks appear to be associated with LTPA [4, 5]. Socio-cultural attributes such as social support, social networks, socioeconomic differences, social cohesion and social capital also seem to shape one's LTPA behaviour [6]. In addition, there is evidence that utility of certain built environment 
features such as parks and sports facilities for recreational purposes was also mediated by social environment [7]. Thus, influence of social and built environment characteristics in the local neighbourhoods would be a possible reason for this low level of LTPA among Sri Lankan adults.

Since people experience or perceive their neighbourhood built environment differently, objective measures of the built environment may not reflect their actual use of the environment $[8,9]$ for PA. From a multi-centre study, Cerin et al. [8] suggested that objective environmental conditions affect PA through one's perceptions, where safety, aesthetics and pedestrian infrastructure are key attributes. Thus, measuring and altering perceptions of people about their neighbourhood environment are more likely to affect cognitions supportive of forming intentions to be physically active $[10,11]$. This is especially true for LTPA, as people are not compelled to carryout PA during their leisure unlike domestic and job-related PA.

Due to the diversity of culture, social-context, infrastructure and policies across countries [12, 13], each setting would have unique relationships between the environment and LTPA. Similarly, social factors appear to be differently affecting LTPA of Asians who live in their own countries than elsewhere [14]. Thus, additional studies beyond high-income Western and Asian populations living in Western countries are necessary to improve the generalizability of available findings and to facilitate cross-country comparisons.

Thus, the aim of this study was to assess the perceived social and built environment associations of leisure-time physical activity among adults living in Colombo District, Sri Lanka.

\section{Main text \\ Methods}

This study was a component of a community based cross sectional study conducted in the district of Colombo among a representative sample of adults aged 20-59 years living in urban and rural areas of Colombo District for a period of more than six months. The sample was selected using stratified cluster sampling probability proportionate to size of adults in a village within the Colombo district. Houses were selected randomly from each cluster followed by selection of an adult randomly. Adults with physical and mental disability or illness preventing engagement in PA, institutionalised adults, pregnant women, as well as adult visitors to the study area were excluded. A full description of the sampling procedure was published elsewhere [3].
Socio-demographic information including age, employment status, level of education, income, sector of residence and gender were assessed using an interviewer administered questionnaire.

Participants self-reported their PA using the long version of International Physical Activity Questionnaire (IPAQ-long) which is culturally adapted and validated in Sri Lanka [15]. IPAQ-long records the number of days and duration of walking, moderate and vigorous intensity PA undertaken during the previous week in different domains (job, transportation, domestic/garden chores and leisure). The PA in leisure time activity was considered for analysis and is described as median and interquartile range along with the mean and standard deviation as the data distribution of LTPA was skewed to the right. Further, the number of persons engaged in any type of LTPA for more than $10 \mathrm{~min}$ was described in different domains of walking, moderate activity, vigorous activity and total activity as numbers and percentages.

The physical and the social environments were assessed using Physical and Social Environment Scale (PASES). The tool was developed and validated to assess the physical and social environment factors associated with PA in Sri Lankan context [16]. This scale measures population density, land use mix diversity, infrastructure for walking, aesthetics and facilities for cycling, vehicular traffic safety, access and connectivity, recreational facilities for PA, safety, social cohesion and social acceptance of PA. Each item in the tool was described using descriptive statistics.

To assess the relationship of LTPA with neighbourhood environment and socio-demographic characteristics a crude analysis was carried out between carrying out any LTPA and the environment attributes. This was followed by a multivariate regression analysis. Gender, sector of residence, education level, monthly income, employment status and self-efficacy were added as covariates in the regression model since they are common covariates of PA in Sri Lankan adults [3]. Self-efficacy was also added as it may affect the perceptions towards the environment [10]. Odds ratios (ORs) with 95\% confidence intervals were calculated and the statistical significance was set at $\mathrm{p}<0.05$. Data analysis was carried out using SPSS -17 package.

All participants responded completely to all questions and there were no missing values as the information was collected using an interviewer administered questionnaire by trained pre-intern medical officers who visited households at a time convenient to the participants, and as the questions were not very sensitive. 


\section{Results}

Most participants were in the low-income category and were currently employed; and the full description of the sample was published previously [3]. Distribution of LTPA among the participants is given in Table 1. Only $21.7 \%$ of adults participated in any LTPA and the commonest type of LTPA was walking, carried out by $14.5 \%$ of sample.

The neighbourhood environment characteristics are presented in Table 2 with a higher score indicating an environment conducive for PA. Items with a median score less than 2 showed the attributes in the environment that were not conducive for PA. They are: non availability of a separate grass/sand strip for walking, pavement obstructions, non-availability of cycle lanes, not having large trees and interesting things to look at, dust and fumes in the environment, not having free or very low cost centers for sports and exercise, not having adequate street lights and a social environment factor of less encouragement for PA from the neighbours.

Table 3 shows that after adjusting for covariates, being male $(\mathrm{OR}=4.36 ; \mathrm{CI}=2.99-6.36)$, living in urban areas $(\mathrm{OR}=1.81 ; \mathrm{CI}=1.11-2.94)$, higher education $(\mathrm{OR}=1.59$; $\mathrm{CI}=1.12-2.25)$, higher income $(\mathrm{OR}=1.61$; $\mathrm{CI}=1.13-$ 2.29 ) and those with higher perceived self-efficacy for PA $(\mathrm{OR}=1.85 ; \mathrm{CI}=1.50-2.28)$ were significantly associated with carrying out some LTPA. Further, social acceptance for PA was associated with higher LTPA $(\mathrm{OR}=2.14$; $\mathrm{CI}=1.65-2.77)$ while age was inversely associated with LTPA. Although, perception of the infrastructure for walking $(\mathrm{OR}=0.44 ; \mathrm{CI}=0.25-0.77)$, and recreational facilities for PA $(\mathrm{OR}=0.80 ; \mathrm{CI}=0.68-0.96)$ showed significant relationship to LTPA, the direction of the association was reversed to what was expected.

\section{Discussion}

This study assessed the perceived social and built environment associations of LTPA among adults living in Colombo District, Sri Lanka. The results revealed that males, urban dwellers, young adults, people with higher education and income have been carrying out some LTPA compared to their counterparts. Although perceptions of infrastructure for walking and recreational facilities were not positively associated with accumulating at least some LTPA, social acceptance and self-efficacy were seen to be positively associated.

Surprisingly, it was found that most of the built environment features were not significantly associated with LTPA, and in particular, perceptions of infrastructure for walking and recreational facilities were inversely related to LTPA. These inverse relationships might be due the fact that people who do at least some LTPA are more aware of the quality, accessibility, condition and usability of available infrastructure and facilities in their neighbourhood, rather than people who are not involve in any LTPA. Further, poor satisfaction of available infrastructure and facilities might limit participation in LTPA [4] and drive people to use facilities far from their neighbourhoods. Supporting this, studies have also shown that apart from poor conditions, amenities [17], location [18] and perceived incivilities in the community also directly hinder utility of public recreational facilities [19]. Thus, importance of maintaining quality of recreational facilities and designing facilities complying with specific needs and recreational preferences of a community are emphasized. Sri Lankans are more active in domestic and job-related PA [3] for which built environment is less relevant, and this may be another reason for the observed poor relationship between built environment and LTPA. Our findings are in contrast to evidence from Western countries [20] and other Asian countries [21, 22]. Our findings suggest that built environment features in typical activity-friendly environments alone do not motivate LTPA of Sri Lankan adults, possibly owing to the unique socio-cultural elements that intervene in making a person active at leisure.

In contrast to Western cultures where LTPA has been increasing [23], social acceptance for LTPA is normally low in South Asian countries where people count regular household chores, caring of children and manual labour as physical exercise [24-26]. As a result, LTPA has not been seen as a requirement in South Asian communities and a majority do not feel motivated to be active during their leisure [27]. This belief may be related to sociocultural background in South Asia [2], or may relate to lack of knowledge about the benefits of LTPA [19, 26]. In some South Asian cultures, spending time for LTPA was considered as selfish [28, 29], while doing outdoor activities, regular exercise and attending gyms were considered

Table 1 Pattern of Leisure time activity of participants $(N=1320)$

\begin{tabular}{|c|c|c|c|c|}
\hline Activity indicator & Walking & Moderate activity & Vigorous activity & Total Leisure \\
\hline Mean Minutes per week of activity (SD) & $18.7(67.1)$ & $13.6(57.7)$ & $5.9(38.2)$ & $38.2(114.0)$ \\
\hline Median Minutes per week of activity (IQR) & $0(0-0)$ & $0(0-0)$ & $0(0-0)$ & $0(0-0)$ \\
\hline At least some activity $n(\%)$ & $191(14.5)$ & $136(10.3)$ & $52(3.9)$ & $287(21.7)$ \\
\hline
\end{tabular}


Table 2 Perceived neighbourhood environment characteristics

\begin{tabular}{|c|c|c|c|}
\hline \multicolumn{2}{|c|}{ Neighbourhood environment characteristics ( $N=1320)$} & \multirow{2}{*}{$\begin{array}{l}\text { Mean } \\
3.32(1.426)\end{array}$} & \multirow{2}{*}{$\begin{array}{l}\text { Median } \\
4(2-4)\end{array}$} \\
\hline Infrastructure for walking & Sidewalks in the main street [Q1] & & \\
\hline & Grass/sand strip for walking in the by-roads with vehicular traffic [Q2] & $2.23(1.208)$ & $2.0(1-3)$ \\
\hline & Pavements/ sidewalks free of obstruction [Q3] & $2.97(1.418)$ & $2.0(2-4$ \\
\hline & Pavements/ sidewalks free of hazards [Q4] & $3.77(1.208)$ & $4.0(3-5)$ \\
\hline \multirow[t]{4}{*}{ Aesthetics and facilities for cycling } & Special lanes, separated paths to cycle [Q5] & $1.42(0.722)$ & $1.0(1-2)$ \\
\hline & Shade in the pathways [Q6] & $3.32(1.229)$ & $4.0(2-4)$ \\
\hline & Trees in the neighbourhood [Q7] & $3.07(1.233)$ & $3.0(2-4)$ \\
\hline & $\begin{array}{l}\text { Interesting/pleasant things to look (Natural sights, beautiful build- } \\
\text { ings) [Q8] }\end{array}$ & $2.40(1.043)$ & $2.0(2-4)$ \\
\hline \multirow[t]{4}{*}{ Vehicular traffic safety } & Free of dust and fumes [Q14] & $2.83(1.364)$ & $2.0(2-4)$ \\
\hline & Low movement of traffic [Q15] & $3.06(1.243)$ & $4.0(2-4)$ \\
\hline & Low speed of vehicles [16] & $3.15(1.169)$ & $4.0(2-4)$ \\
\hline & Less road traffic accidents [Q20] & $3.49(1.214)$ & $4.0(2-4)$ \\
\hline \multirow[t]{6}{*}{ Access and connectivity } & Alternative routes for getting from place to place [Q9] & $3.70(0.879)$ & $4.0(4-4)$ \\
\hline & $\begin{array}{l}\text { Many places that need to be visited day to day are within easy walking } \\
\text { distance [Q10] }\end{array}$ & $3.65(1.112)$ & $4.0(4-4)$ \\
\hline & Short distance to main road [Q1 1] & $\begin{array}{l}3.82 \\
(1.046)\end{array}$ & $4.0(4-4)$ \\
\hline & Short distance to transit stop/ public transport [Q12] & $3.76(1.050)$ & $4.0(4-4)$ \\
\hline & Terrain is not hilly and good for walking [Q13] & $4.12(1.033)$ & $4.0(4-4)$ \\
\hline & Presence of pedestrian crossing, signals and overhead bridges [Q24] & $3.09(1.244)$ & $4.0(2-4)$ \\
\hline \multirow[t]{3}{*}{ Recreation facilities for PA } & Free or very low cost centres for sports and exercise [Q17] & $2.88(1.137)$ & $3.0(2-4)$ \\
\hline & Public spaces for sport and recreation (Park, beach) [Q18] & $3.15(1.182)$ & $4.0(2-4)$ \\
\hline & Easy access to the public places for sports and recreation [Q19] & $3.11(1.188)$ & $4.0(2-4)$ \\
\hline \multirow[t]{3}{*}{ Safety } & Low crime rate[Q21] & $3.35(1.205)$ & $4.0(2-4)$ \\
\hline & Well-lit roads [Q22] & $2.61(1.173)$ & $2.0(2-4)$ \\
\hline & Free of stray animals [Q23] & $3.26(1.179)$ & $4.0(2-4)$ \\
\hline \multirow[t]{6}{*}{ Social cohesion } & Harmony between people in the neighbourhood [Q25] & $3.68(0.948)$ & $4.0(3-4)$ \\
\hline & Respect among persons in the neighbourhood [Q26] & $3.46(0.965)$ & $4.0(3-4)$ \\
\hline & Free of social disorder/ social disputes [Q27] & $3.57(0.889)$ & $4.0(3-4)$ \\
\hline & Helpful people in the neighbourhood [Q28] & $3.83(0.745)$ & $4.0(4-4)$ \\
\hline & Trustworthy people in the neighbourhood [Q29] & $3.43(0.927)$ & $4.0(3-4)$ \\
\hline & Good interactions between people in the neighbourhood [Q30] & $3.28(1.016)$ & $4.0(2-4)$ \\
\hline \multirow[t]{4}{*}{ Social acceptance of PA } & People in the neighbourhood physically active [Q31] & $2.86(1.134)$ & $3.0(2-4)$ \\
\hline & The people encourage each other for active living [Q32] & $2.32(0.940)$ & $2.0(2-3)$ \\
\hline & Social acceptance for being active for day to day activities [Q33] & $3.20(0.997)$ & $3.0(2-4)$ \\
\hline & social acceptance for walking, exercising and recreational PA [Q34] & $2.87(1.004)$ & $3.0(2-4)$ \\
\hline \multicolumn{2}{|c|}{ Composite land use mix and accessibility score } & $3.33(0.813)$ & $3.36(2.8-4$ \\
\hline
\end{tabular}

weird [24-26]. Thus, in most South Asian cultures, including the current study sample, the society encourages people to be active within day-to-day activities [25, $26,28]$. However, it was seen in the present study that the odds of having some LTPA was significantly higher with those who perceived having higher social acceptance for LTPA in their societies. Further social acceptance was observed as the most influential correlate of LTPA in our sample. Strong family, societal, and religious ties observed among the Sri Lankans may be the reason for the requirement of social acceptance to involve in LTPA, which otherwise may be considered as culturally inappropriate behaviour. Thus, LTPA promotion efforts in Sri Lanka should focus on gaining social acceptance for LTPA, which would mitigate social barriers such as feeling embarrassed of being seen by others while exercising in public places $[26,27]$, wearing specific garments while performing exercise [28] and judged negatively by others at recreational places [19]. 
Table 3 Leisure time physical activity and its association with socio-demographic and environment characteristics

\begin{tabular}{|c|c|c|}
\hline & Unadjusted Odds ratio $(95 \% \mathrm{Cl})$ & Adjusted Odds ratio $(95 \% \mathrm{Cl})$ \\
\hline \multicolumn{3}{|c|}{ Environment and socio-demographic characteristics } \\
\hline Infrastructure for walking & $\begin{array}{l}0.42^{*} \\
(0.26-0.68)\end{array}$ & $\begin{array}{l}0.44^{*} \\
(0.25-0.77)\end{array}$ \\
\hline Aesthetics and facilities for walking & $\begin{array}{l}1.26^{*} \\
(1.04-1.53)\end{array}$ & $\begin{array}{l}0.87 \\
(0.66-1.13)\end{array}$ \\
\hline Vehicular traffic safety & $\begin{array}{l}0.90 \\
(0.79-1.02)\end{array}$ & $\begin{array}{l}1.14 \\
(0.90-1.44)\end{array}$ \\
\hline Access and connectivity & $\begin{array}{l}0.89 \\
(0.73-1.07)\end{array}$ & $\begin{array}{l}0.91 \\
(0.67-1.22)\end{array}$ \\
\hline Recreation facilities for PA & $\begin{array}{l}0.79^{*} \\
(0.69-0.90)\end{array}$ & $\begin{array}{l}0.80^{*} \\
(0.68-0.96)\end{array}$ \\
\hline Safety & $\begin{array}{l}0.79^{*} \\
(0.68-0.92)\end{array}$ & $\begin{array}{l}0.87 \\
(0.70-1.09)\end{array}$ \\
\hline Social cohesion & $\begin{array}{l}1.36^{*} \\
(1.07-1.74)\end{array}$ & $\begin{array}{l}0.99 \\
(0.73-1.33)\end{array}$ \\
\hline Social acceptance of PA & $\begin{array}{l}1.97^{*} \\
(1.62-2.40)\end{array}$ & $\begin{array}{l}2.14^{*} \\
(1.65-2.77)\end{array}$ \\
\hline Land use mix score & $\begin{array}{l}0.96 \\
(0.82-1.13)\end{array}$ & $\begin{array}{l}1.23 \\
(0.95-1.59)\end{array}$ \\
\hline \multicolumn{3}{|l|}{ Residential density } \\
\hline Annexed/ row houses/2-3 story flats & $\begin{array}{l}0.68 \\
(0.49-0.95)\end{array}$ & $\begin{array}{l}0.67 \\
(0.40-1.12)\end{array}$ \\
\hline Mix of detached and annex houses & $\begin{array}{l}1.72^{*} \\
(1.15-2.59)\end{array}$ & $\begin{array}{l}1.41^{*} \\
(0.81-2.46)\end{array}$ \\
\hline Apartment 4-stories or above & $\begin{array}{l}0.61 \\
(0.23-1.60)\end{array}$ & $\begin{array}{l}1.29 \\
(0.37-4.45)\end{array}$ \\
\hline Detached single family & Reference & Reference \\
\hline Age in years & $\begin{array}{l}0.98^{*} \\
(0.97-0.99)\end{array}$ & $\begin{array}{l}0.97^{*} \\
(0.96-0.98)\end{array}$ \\
\hline \multicolumn{3}{|l|}{ Gender } \\
\hline Male & $\begin{array}{l}3.70^{*} \\
(2.78-4.92)\end{array}$ & $\begin{array}{l}4.36^{*} \\
(2.99-6.36)\end{array}$ \\
\hline Female & Reference & Reference \\
\hline \multicolumn{3}{|l|}{ Sector of residence } \\
\hline Urban & $\begin{array}{l}2.00^{*} \\
(1.43-2.81)\end{array}$ & $\begin{array}{l}1.81^{*} \\
(1.11-2.94)\end{array}$ \\
\hline Rural & $\begin{array}{l}1.74^{*} \\
(1.20-2.52)\end{array}$ & $\begin{array}{l}1.32 \\
(0.74-2.37)\end{array}$ \\
\hline CMC & Reference & Reference \\
\hline \multicolumn{3}{|l|}{ Education Level } \\
\hline Passed G.C.E A/L or higher & $\begin{array}{l}2.48^{*} \\
(1.89-3.25)\end{array}$ & $\begin{array}{l}1.59^{*} \\
(1.12-2.25)\end{array}$ \\
\hline Education less than G.C.E. A/L & Reference & Reference \\
\hline \multicolumn{3}{|l|}{ Average monthly income in Rupees (USD) } \\
\hline$>30,000(230)$ & $\begin{array}{l}2.39^{*} \\
(1.81-3.16)\end{array}$ & $\begin{array}{l}1.61^{*} \\
(1.13-2.29)\end{array}$ \\
\hline$<=30,000(>230)$ & Reference & Reference \\
\hline \multicolumn{3}{|l|}{ Work status } \\
\hline Employed & $\begin{array}{l}2.41 \\
(1.81-3.20)\end{array}$ & $\begin{array}{l}0.78 \\
(0.53-1.15)\end{array}$ \\
\hline Not employed & Reference & Reference \\
\hline Self-efficacy score & $\begin{array}{l}2.01^{*} \\
(1.70-2.42)\end{array}$ & $\begin{array}{l}1.85^{*} \\
(1.50-2.28)\end{array}$ \\
\hline Constant & & 0.14 \\
\hline
\end{tabular}


Self-efficacy has been identified as a strong predictor of leisure time exercise behaviour in adults [30]. Selfefficacy is a form of cognitively based motivation that is described as the confidence in one's own ability to successfully execute a particular behavior [31]. The choice of behavior, amount of effort, and sustainability are depend on the person's self-efficacy that relies on performance accomplishment, vicarious experience, verbal persuasion and emotional arousal [31]. Self-efficacy also facilitates initiating and continuing exercise [32]. For example, selfefficacy was a predictor of initiating PA among women with gestational diabetes mellitus who received obstetric care at a teaching hospital in Sri Lanka [33]. Studies have also shown that self-efficacy mitigates the constraints of living in poor walkable environments, towards being physically active at leisure [34, 35]. Although self-efficacy has been widely acknowledged as a critical factor of promoting LTPA in Western countries [30, 35, 36], this is one of the few to demonstrate that self-efficacy is a positive correlate of LTPA among Sri Lankan adults. The findings of the current study comply with Social Learning Theory [31]. Collectively, the results of this study suggest that interventions improving LTPA should primarily focus on improving self-efficacy. Infrastructure and recreational facilities might be less important to people if the people are motivated to carry out LTPA.

Social acceptance and self-efficacy can be considered the most influential factors for facilitating LTPA among Sri Lankan adults. Although the perception of built environment features were not positively associated, it might be interesting to look also at the objective measurements together at the same time to understand these complex relationships. However, the findings open up the discussion of built environment influences to PA in a South Asian setting.

\section{Limitations}

Self-reported questionnaire was used to quantify PA, which may be affected by recall and social desirability bias. The sample was drawn from only one district in Sri Lanka which further precludes the generalizability of the results. Additionally, the cross-sectional nature of our study cannot prove a causal association between neighbourhood environment and LTPA.

\section{Abbreviations \\ IPAQ: International Physical Activity Questionnaire; LTPA: Leisure-time physical activity; NCDs: Non-communicable diseases; PA: Physical activity.}

\section{Acknowledgements}

Authors wish to thank the administrators of the District of Colombo, and the participants of the study for their support and participation.

\section{Authors' contributions}

SDW-contributed to the design, planning the study, literature search, acquisition of data, analysis and interpretation of the results, drafting the manuscript and revising it critically and has given final approval of the version to be published. MP -contributed to literature search, drafting the manuscript, drawing conclusions and in revising it critically for important intellectual content and has given final approval of the version to be published. JG—contributed to the planning of the study, drawing conclusions and in revising it critically for important intellectual content the preparation of the research paper and has given final approval of the version to be published. All authors have given consent for publication. All authors read and approved the final manuscript.

\section{Funding}

The University Grants Commission of Sri Lanka funded this research (Grant No UGC/ICD/CRF2009), where funding was for primary data collection.

\section{Availability of data and materials}

The datasets used and/or analysed during the current study are available from the corresponding author on reasonable request.

\section{Declarations}

\section{Ethics approval and consent to participate}

The Ethics Review Committee of Faculty of Medicine, University of Colombo approved the study protocol (reference number EC-09-084) and data collection was carried out after obtaining informed written consent from each participant.

\section{Consent for publication}

Not applicable as no individual personal data in any form is presented here.

\section{Competing interests}

None of the author has any financial interest or received any financial benefit from this research.

\section{Author details}

'Department of Community Medicine, University of Colombo, Colombo, Sri Lanka. ${ }^{2}$ Department of Gynaecology and Obstetrics, Faculty of Medicine and Allied Sciences, Rajarata University of Sri Lanka, Anuradhapura, Sri Lanka. ${ }^{3}$ Postgraduate Institute of Science, University of Peradeniya, Peradeniya, Sri Lanka. ${ }^{4}$ Department of Geology, University of Peradeniya, Peradeniya, Sri Lanka.

Received: 10 June 2021 Accepted: 12 October 2021

Published online: 20 October 2021

\section{References}

1. Lee I-M, Shiroma EJ, Lobelo F, Puska P, Blair SN, Katzmarzyk PT, et al. Effect of physical inactivity on major non-communicable diseases worldwide: an analysis of burden of disease and life expectancy. Lancet. 2012:380:219-29.

2. Ranasinghe $C D$, Ranasinghe $P$, Jayawardena R, Misra A. Physical activity patterns among South-Asian adults: a systematic review. Int J Behav Nutr Phys Act. 2013;10:116

3. De Silva WS, Fernando D, Gunatilake J. Pattern of physical activity among Sri Lankan adults in the District of Colombo: a cross-sectional study. Asia Pac J Public Health. 2016;28:725-36.

4. Sallis JF, Cervero RB, Ascher W, Henderson KA, Kraft MK, Kerr J. An ecological approach to creating active living communities. Annu Rev Public Health. 2006:27:297-322.

5. Sallis JF, Floyd MF, Rodríguez DA, Saelens BE. Role of built environments in physical activity, obesity, and cardiovascular disease. Circulation. 2012;125:729-37.

6. Lindström M, Hanson BS, Östergren PO. Socioeconomic differences in leisure-time physical activity: the role of social participation and social capital in shaping health related behaviour. Soc Sci Med. 2001:52:441-51.

7. Jun HJ, Park S. The effect of cross-level interaction between community factors and social capital among individuals on physical 
activity: considering gender difference. Int J Environ Res Public Health. 2019;16:495.

8. Cerin E, Conway TL, Adams MA, Barnett A, Cain KL, Owen N, et al. Objectively-assessed neighbourhood destination accessibility and physical activity in adults from 10 countries: an analysis of moderators and perceptions as mediators. Soc Sci Med. 2018;211:282-93.

9. Jia X, Yu Y, Xia W, Masri S, Sami M, Hu Z, et al. Cardiovascular diseases in middle aged and older adults in China: the joint effects and mediation of different types of physical exercise and neighborhood greenness and walkability. Environ Res. 2018;167:175-83.

10. Gebel K, Bauman A, Owen N. Correlates of non-concordance between perceived and objective measures of walkability. Ann Behav Med. 2009:37:228-38

11. McCormack GR, Spence JC, Berry T, Doyle-Baker PK. Does perceived behavioral control mediate the association between perceptions of neighborhood walkability and moderate-and vigorous-intensity leisuretime physical activity? J Phys Act Health. 2009;6:657-66.

12. Sallis JF, Bowles HR, Bauman A, Ainsworth BE, Bull FC, Craig CL, et al. Neighborhood Environments and physical activity among adults in 11 countries. Am J Prev Med. 2009;36:484-90.

13. Chen TA, Lee JS, Kawakubo K, Watanabe E, Mori K, Kitaike T, et al. Features of perceived neighborhood environment associated with daily walking time or habitual exercise: differences across gender, age, and employment status in a community-dwelling population of Japan. Environ Health Prev Med. 2013;18:368-76.

14. Yi SS, Trinh-Shevrin C, Yen IH, Kwon SC. Racial/ethnic differences in associations between neighborhood social cohesion and meeting physical activity guidelines, United States, 2013-2014. Prev Chronic Dis. 2016;13:E165

15. Karunapema RP. Evaluation of the effectiveness of a lifestyle intervention in primary prevention of diabetes among adults with impaired fasting glucose from a rural area in Gampaha District. Sri Lanka: University of Colombo; 2007. http://archive.cmb.ac.lk/research/handle/70130/1038

16. De Silva Weliange SH, Fernando D, Gunatilake J. Development and validation of a tool to assess the physical and social environment associated with physical activity among adults in Sri Lanka. BMC Public Health. 2014;14:423.

17. Reynolds KD, Wolch J, Byrne J, Chou CP, Feng G, Weaver S, et al. Trail characteristics as correlates of urban trail use. Am J Health Promot. 2007;21(Suppl 4):335-45.

18. Pussella PG, Li L. Identification and assessment of the driving forces for the use of urban green parks and their accessibility in Colombo, Sri Lanka, through analytical hierarchical processing. Geospat Health. 2019;14:72-80.

19. De Silva WS, Fernando D, Gunatilake J. Neighbourhood social environment on physical activity among adults in Sri Lanka: a qualitative inquiry. J Col Commun Phys Sri Lanka. 2018;24:28-35.

20. Van Dyck D, Cerin E, Conway TL, De Bourdeaudhuij I, Owen N, Kerr J, et al. Perceived neighborhood environmental attributes associated with adults' leisure-time physical activity: findings from Belgium Australia and the USA. Health Place. 2013:19:59-68.

21. Hanibuchi T, Nakaya T, Yonejima M, Honjo K. Perceived and objective measures of neighborhood walkability and physical activity among adults in Japan: a multilevel analysis of a nationally representative sample. Int J Environ Res Public Health. 2015:12:13350-64.
22. Jia Y, Usagawa T, Fu H. The association between walking and perceived environment in Chinese community residents: a cross-sectional study. PLOS ONE. 2014;9:e90078.

23. López-Bueno R, Smith L, Tully MA, Shin J, Calatayud J, López-Sánchez GF, et al. Increase in regular leisure-time physical activity in Spanish adults between 1987 and 2017. Am J Prev Med. 2021;61:e73-9.

24. Mathews E, Lakshmi JK, Ravindran TKS, Pratt M, Thankappan KR. Perceptions of barriers and facilitators in physical activity participation among women in Thiruvananthapuram City. India Glob Health Promot. 2016;23:27-36

25. Kadariya S, Aro AR. Barriers and facilitators to physical activity among urban residents with diabetes in Nepal. PLoS ONE. 2018;13:e0199329.

26. Medagama A, Galgomuwa M. Lack of infrastructure, social and cultural factors limit physical activity among patients with type 2 diabetes in rural Sri Lanka, a qualitative study. PLoS ONE. 2018;13:e0192679.

27. Karunanayake AL, Senaratne CD, Stathi A. A descriptive cross sectional study comparing barriers and determinants of physical activity of Sri Lankan middle aged and older adults. PLoS ONE. 2020;15:e0232956.

28. Lawton J, Ahmad N, Hanna L, Douglas M, Hallowell N. "I can't do any serious exercise": barriers to physical activity amongst people of Pakistani and Indian origin with type 2 diabetes. Health Educ Res. 2006;21:43-54.

29. Sriskantharajah J, Kai J. Promoting physical activity among South Asian women with coronary heart disease and diabetes: what might help? Fam Pract. 2007:24:71-6.

30. Hovell MF, Sallis JF, Hofstetter CR, Spry VM, Faucher P, Caspersen CJ. Identifying correlates of walking for exercise: an epidemiologic prerequisite for physical activity promotion. Prev Med. 1989;18:856-66.

31. Bandura A. Self-efficacy: toward a unifying theory of behavioral change Psychol Rev. 1977:84:191-215.

32. Sallis JF, Hovell MF, Hofstetter CR. Predictors of adoption and maintenance of vigorous physical activity in men and women. Prev Med. 1992;21:237-51.

33. Pinidiyapathirage J, Jayasuriya R, Cheung NW, Schwarzer R. Self-efficacy and planning strategies can improve physical activity levels in women with a recent history of gestational diabetes mellitus. Psychol Heal. 2018:33:1062-77.

34. Kaczynski AT, Robertson-Wilson J, Decloe M. Interaction of perceived neighborhood walkability and self-efficacy on physical activity. J Phys Act Health. 2012;9:208-17.

35. Bengoechea EG, Spence JC, McGannon KR. Gender differences in perceived environmental correlates of physical activity. Int J Behav Nutr Phys Act. 2005;2:12

36. Cerin E, Leslie E. How socio-economic status contributes to participation in leisure-time physical activity. Soc Sci Med. 2008;66:2596-609.

\section{Publisher's Note}

Springer Nature remains neutral with regard to jurisdictional claims in published maps and institutional affiliations. 